\title{
Correction: Anti-prothrombin autoantibodies enriched after infection with SARS-CoV-2 and influenced by strength of antibody response against SARS-CoV-2 proteins
}

\author{
Marc Emmenegger, Sreedhar Saseendran Kumar, Vishalini Emmenegger, \\ Tomas Malinauskas, Thomas Buettner, Laura Rose, Peter Schierack, Martin F. Sprinzl, \\ Clemens J. Sommer, Karl J. Lackner, Adriano Aguzzi, Dirk Roggenbuck, Katrin B. \\ M. Frauenknecht
}

The order of S1 Fig, S3 Fig, and S4 Fig is incorrectly switched. The file that appears as S1 Fig should be S3 Fig, the file that appears as S3 Fig should be S4 Fig, and the file that appears as S4 Fig should be S1 Fig. The Supporting Information captions appear in the correct order.

\section{Supporting information}

S1 Fig. Demographic features of non-infected controls and SARS-CoV-2 infected individuals. A. Age distribution of entire cohort for non-infected controls (orange) and SARS-CoV-2 infected individuals (blue). The distribution of the controls indicates a generally younger age versus the SARS-CoV-2 infected individuals. B. Sex distribution of entire cohort for noninfected controls (orange) and SARS-CoV-2 infected individuals (blue). The distribution in both cohorts was slightly skewed towards males versus females. C. Female (violet) and male (light orange) representation (in \%) within each severity score. The distributions are slightly skewed towards more male than female individuals in all severity scores, except for score 2 . D. Representation of DPO related to severity score. The convalescent individuals included in this study and sampled at later DPO all had a severity score of 1 while the acutely infected hospital patients had severity scores between $2-4$.

(TIF)

S3 Fig. TRABI comparison of serum and plasma and UMAP representation of SARS-CoV2 IgG profiles. A. Binding curves of patient-matched serum and plasma samples. Three SARS-CoV-2 proteins were used to conduct the tripartite-autoimmune blood immunoassay technology: the SARS-CoV-2 spike ectodomain (S), its receptor binding domain (RBD), and the nucleocapsid protein (NC). All samples were tested as technical duplicates and are shown as mean with standard deviation. B. Based on the binding curves, the concentration at halfmaximum binding of the sigmoidal curve, or the $\mathrm{p}(\mathrm{EC} 50)$ value, was calculated. $\mathrm{p}(\mathrm{EC} 50)$ values for $\mathrm{S}, \mathrm{RBD}$, and $\mathrm{NC}$ for all patients are compared in a scatter plot with serum-based $\mathrm{p}$ (EC50) values on the $\mathrm{x}$-axis and plasma-based $\mathrm{p}$ (EC50) values on the $\mathrm{y}$-axis. The Pearson correlation coefficient was 0.9942 (95\% confidence interval: $0.9865-0.9975)$ and $\mathrm{R}^{2}$ was 0.9885 . Dotted line represents the $95 \%$ confidence interval of the linear regression. C. Visualisation of the same data as shown in (B) using a Bland-Altman plot. Bias was calculated to be 0.01976 with a standard deviation of 0.1361 . Dotted lines represent the confidence intervals $(-0.2471$ to 0.2866). D. UMAP representation of anti-SARS-CoV-2 protein antibodies displays clear 
clusters, with non-infected controls (black) and non-IgG-reactive SARS-CoV-2 infected individuals (turquoise) clustering separately from IgG-reactive SARS-CoV-2 infected individuals. (TIF)

S4 Fig. Age, severity, and DPO in relation to PT and $\beta 2$ IgM levels. A. Severity score as a function of age, with colour-coded PT (left) and $\beta 2$ (right) IgM levels. When ignoring the interdependency of features, data suggests that PT and $\beta 2$ IgM levels are modulated by severity but not by age. B. PT and $\beta 2$ IgM levels as a function of DPO. PT IgM aPL levels show a nonhomogenous distribution along DPO, with a rise between DPO 10-30 and a subsequent drop. $\beta 2$ IgM levels, on the other hand, seem stable over time. To summarize the data, aPL levels were binned in 15 day windows. The LMM used in this study takes care of the interdependency of parameters and accounts for redundancy, in the present case e.g., between severity score and DPO and their effect on PT or $\beta 2$ IgM titres. Values are shown as single dots, with boxplot (median and interquartile range) in (A) and as mean \pm standard deviation in (B). (TIF)

\section{Reference}

1. Emmenegger M, Kumar SS, Emmenegger V, Malinauskas T, Buettner T, Rose L, et al. (2021) Anti-prothrombin autoantibodies enriched after infection with SARS-CoV-2 and influenced by strength of antibody response against SARS-CoV-2 proteins. PLoS Pathog 17(12): e1010118. https://doi.org/10. 1371/journal.ppat.1010118 PMID: 34860860 